\title{
The influence of the SARS-CoV-2 pandemic on oral and maxillofacial surgery: a nationwide survey among 54 hospitals and 240 private practices in Germany
}

\author{
Andreas Pabst ${ }^{1}$ (1) $\cdot$ Alexander-N. Zeller ${ }^{2} \cdot$ Robert Sader $^{3} \cdot$ Jörg-Ulf Wiegner $^{4} \cdot$ Matthias Schneider $^{5}$. \\ Michael Ehrenfeld ${ }^{6}$. Jürgen Hoffmann ${ }^{7}$
}

Received: 13 August 2020 / Accepted: 25 November 2020 / Published online: 6 January 2021

(C) The Author(s), under exclusive licence to Springer-Verlag GmbH, DE part of Springer Nature 2021

\begin{abstract}
Objective The severe acute respiratory syndrome coronavirus-2 (SARS-CoV-2) pandemic has created hitherto unknown challenges for healthcare systems and patient care. This study aimed to analyze its influence on patient care and healthcare management in oral and maxillofacial surgery (OMFS) in Germany.

Materials and methods A nationwide survey of the German Association of Oral and Maxillofacial Surgery was performed. Individual questionnaires containing 10 questions for university as well as non-university hospitals and 15 questions for private practices (PPs) for OMFS were created to collect data for patient numbers and surgical procedures conducted, usage of personal protection equipment (PPE), SARS-CoV-2 tests, and economic aspects.

Results Fifty-four hospitals and 240 PPs participated in the study. The reduction in ward capacities and number of surgical procedures ranged from 17 to $78 \%$. PPE consisted of standard surgical masks (58\% hospitals, $64 \%$ PPs) and FFP2/N95/KN95 respirators (45\% hospitals, 48\% PPs). Preoperative SARS-CoV-2 tests were more frequently performed in hospitals (34\% vs $2 \%$ ). At PPs, turnover for medical and dental procedures billed to the public insurance had reduced to $58.81 \%$ and $62.43 \%$, respectively, of the corresponding values for 2019 , and $58.75 \%$ of the PPs had applied for short-time allowances.

Conclusion With the exception of some elective procedures, primary patient care in OMFS has been assured during the pandemic. However, the immense economic burden on hospitals and PPs cannot be conclusively assessed at this point.

Clinical relevance For OMFS, the German healthcare system has shown the ability to adapt to emergency situations such as the SARS-CoV-2 pandemic. Adequate surgical capacities in OMFS are necessary even during pandemics.
\end{abstract}

Keywords Coronavirus $\cdot$ Economic burden $\cdot$ Hygiene measures $\cdot$ Patient care $\cdot$ Personal protection equipment

Andreas Pabst and Alexander-N. Zeller contributed equally to this work.

Andreas Pabst

andipabst@me.com

1 Department of Oral and Maxillofacial Surgery, Federal Armed Forces Hospital, Rübenacherstr. 170, 56072 Koblenz, Germany

2 Department of Oral and Maxillofacial Surgery, Hannover Medical School, Carl-Neuberg-Str. 1, 30625 Hannover, Germany

3 Department of Oral, Cranio Maxillofacial and Facial Plastic Surgery, University Hospital Frankfurt, Theodor-Stern-Kai 7, 60528 Frankfurt am Main, Germany
4 Private Practice for Oral and Maxillofacial Surgery, Saalstraße 35, 07318 Saalfeld, Germany

5 Private Practice for Oral and Maxillofacial Surgery, Dr.-Külz-Ring 15, 01067 Dresden, Germany

6 Department of Oral and Maxillofacial Surgery, University Hospital Munich, Lindwurmstr. 2a, 80337 Munich, Germany

7 Department of Oral and Maxillofacial Surgery, University Hospital Heidelberg, Im Neuenheimer Feld 400, 69120 Heidelberg, Germany 


\section{Introduction}

The severe acute respiratory syndrome coronavirus-2 (SARSCoV-2) caused a global pandemic that first broke out in the province of Wuhan (China) in 2019 [1]. The World Health Organization (WHO) declared it a global pandemic on March 11, 2020 [2]. Patients infected with SARS-CoV-2 typically show lung-related symptoms that manifest as severe inflammatory responses and lung injury. The disease, which is referred to as corona virus disease 2019 (COVID-19), can be fatal when it occurs with severe pneumonia [3]. The relevant risk factors that can negatively influence the outcome of COVID-19 included, but is not limited to, male sex, age over 65 years, smoking, and different comorbidities, such as cardiovascular and respiratory diseases [4]. SARS-CoV-2 can cause severe vascular and endothelial damage with widespread thrombosis and microangiopathy in the lungs of affected patients [5]. However, the exact pathological mechanisms in the lung have not yet been fully elucidated.

The SARS-CoV-2 pandemic has created enormous challenges related to all aspects of pandemic management and patient care for healthcare systems worldwide. On the one hand, it is necessary to treat patients with SARS-CoV-2 infection, especially those with severe courses of the infection. This has brought some healthcare systems to the edge of decompensation [6]. On the other hand, there is also a need to continue providing medical care to non-infected in- and outpatients in hospitals and private practices of all medical specialties and to protect them and the medical staff from SARSCoV-2 infection. As of mid-March 2020, the German government asked all public and private hospitals to postpone elective procedures to ensure increased intensive care capacities. By mid-April, hospitals were encouraged by the federal ministry of health to reschedule these postponed appointments to be conducted from the beginning of May ${ }^{1}$. However, the instructions for private practices were, to some degree, contradictory and differed significantly depending on the specific federal state authority.

By April 2020, many private practices were registered as SARS-CoV-19 specialized practices. These registered practices were supposed to act as the first point of contact for SARS-CoV-2-positive patients. They were equipped with additional personal protection equipment (PPE) and were able to bill certain SARS-CoV-2-related surcharges to the statutory health insurance.

Dentists, oral and maxillofacial surgeons, and ear-nosethroat (ENT) specialists treating patients with SARS-CoV-2 infection were assumed to be at a high risk of getting infected themselves. This was mainly attributed to their proximity to

\footnotetext{
${ }^{1}$ https://www.aerzteblatt.de/nachrichten/112048/Spahn-SchrittweiseRueckkehr-in-einen-Regelbetrieb-in-Kliniken; source last assessed online on July 18,2020
}

the respiratory tract and the oral cavity, and the presence of SARS-CoV-2 in the saliva and nasal secretions of infected patients [7-9]. This risk may be increased by the use of medical instruments that can create potentially contagious droplets and aerosols, such as $\mathrm{CO}_{2}$ lasers, electrosurgical units, and rotating devices such as drills and saws [10-12]. In this context, SARS-CoV-2 infections in oral and maxillofacial surgical residents and the resultant mortalities have been reported [2]. Consequently, implementation of protection protocols and increased use of PPE for medical staff were necessary to reduce the risk of infections among healthcare workers. Increased rates of SARS-CoV-2 infections among healthcare workers and medical staff can further increase the burden on and the limitations of the healthcare system [12-15]. Thus, new management strategies, such as telephone hotlines and video consultations, were established, with the aim of decreasing physical contact with patients during the pandemic [16-18]. The insufficient availability of PPE also led to the development of various innovative and partly improvised solutions using modern technologies such as $3 \mathrm{D}$ printing, which is traditionally well represented in the field of oral and maxillofacial surgery, e.g., the widespread use of 3D-printed face shields $[19,20]$.

To conclude, oral and maxillofacial surgeons at (non-) university hospitals and private practices in Germany faced a multitude of problems during the pandemic. On the one hand side, they experienced difficulties related to aspects such as patient care, infection protection and hygiene, and the availability of PPE and SARS-CoV-2 tests. On the other hand, the pandemic created newer economic challenges due to the significantly decreased patient numbers and the lack of governmental financial and non-financial support. To understand these challenges in greater detail, this study aimed to identify the influences of the SARS-CoV-2 pandemic on hospitals and private practices (including ambulatory health care centers and those holding inpatient beds), and to analyze the medical and economic burden caused by the SARS-CoV-2 pandemic on patient care and healthcare management in all fields of oral and maxillofacial surgery (OMFS) in Germany.

\section{Material and methods}

A nationwide online survey was performed by the German Association of Oral and Maxillofacial Surgery (DGMKG). Two separate questionnaires, including 10 questions for participants working at university and non-university hospitals and 15 questions for those working at private practices for oral and maxillofacial surgery were created using SurveyMonkey (San Mateo, CA, USA). Due to the differences in regulations, official recommendations, and the surgical spectrum, separate questionnaires were designed for private practices and hospitals. The questionnaires were kept concise to achieve the 
highest completion rate possible and to obtain specific information from these two cohorts. In addition to basic data and key economic data, the surveys contained questions about measures taken to adapt to the new situation. Furthermore, PPE supply and PPE use in general and changes in the frequency of surgical procedures were evaluated. Table 1 illustrates the contents of the questionnaires for participants from hospitals and private practices in detail. An internal validation of the questionnaires was performed by the DGMKG board prior to the start of the survey. For this purpose, the number of questions, their length, and their order were evaluated automatically. Consequently, the questionnaire was distributed among DGMKG board members and modified according to the suggestions of the test participants.

In total, 79 heads of departments of oral and maxillofacial surgery in hospitals and 935 oral and maxillofacial surgeons working in private practices in Germany were contacted via email by the board and head office of the DGMKG (Hofheim, Germany). They were invited to take part in this survey on an anonymous basis. Responses were limited to one reply per hospital and one reply per private practice. The surveys were conducted between April 29 and June 24, 2020. Results were collected using SurveyMonkey, analyzed using Wizard for Mac 1.9.42 by Evan Miller, and illustrated in numbers (Apple, Cupertino, CA, USA). For descriptive statistics, mean values and standard deviations (in brackets, after a plus-minus sign) are given. The distribution of dichotomous variables was evaluated using the chi-squared test (equivalent to $z$-score).

\section{Results}

Completed questionnaires were obtained from 240 private practices and 54 hospitals (27 university hospitals and 27 nonuniversity hospitals). The response rate was $68.35 \%$ for hospitals and $25.59 \%$ for private practices. Within the university- and non-university hospitals, the ward capacity used reduced by $43.20 \%$ ( $\pm 25.75 \%)$ due to the pandemic, while the frequency of inpatient surgical procedures reduced by $47.58 \%$ ( \pm $21.10 \%$ ). The number of outpatient surgical procedures under general anesthesia reduced by $77.59 \%( \pm 21.45 \%)$ and those under local anesthesia reduced by $70.33 \%( \pm 20.97 \%)$. In private practices, the frequency of inpatient surgical procedures reduced by $40.47 \%$ ( $\pm 43.34 \%$ ). In general, $20.68 \%$ of the private practices stated that they did have beds for inpatients available. Their number of outpatients reduced by $45.31 \%$ ( \pm $24.27 \%$ ), and the number of outpatient surgical procedures under general anesthesia reduced by $44.18 \%( \pm 35.68 \%)$, while the number of those outpatient procedures under local anesthesia reduced by $49.74 \%( \pm 26.26 \%)$.

In the private practice, screening for SARS-CoV-19 (Fig. 1) was conducted once preoperatively by $5.33 \%$ and twice by $1.78 \%$ of the participants, while $93.78 \%$ stated that they did not routinely conduct preoperative tests for SARSCoV-19. Within the university and non-university hospitals, $33.96 \%$ of the participants stated that they routinely conducted a SARS-CoV-19 test once preoperatively, $37.74 \%$ stated that they conducted a SARS-CoV-19 test before admission to the ward, and $3.77 \%$ stated that they conducted a test after discharge. Most (45.28\%) participants from hospitals stated that they did not routinely conduct a SARS-CoV-19 screening test.

Routinely used personal protective equipment (PPE, Fig. 2) consisted of standard surgical masks in $57.41 \%$ of the university and non-university hospitals, and $64.14 \%$ of the private practices. FFP2 and equivalent (N95 or KN95) respirators were used by $46.30 \%$ of the university and non-university hospitals, and $48.52 \%$ of the private practices. Higher grades of filtering were used by $1.85 \%$ of the hospitals and $6.33 \%$ of the private practices. 3D-printed face shields were used in $22.22 \%$ of hospitals and in $36.71 \%$ of the private practices.

The supply of PPE by the Federal Associations of Statutory Health Insurance Physicians was rated as sufficient by $40.91 \%$ of the SARS-CoV-19-specialized practices and by $39.43 \%$ of the other non-specialized private practices. The supply by the Federal Associations of Sick Fund Dentists was rated as sufficient by $40.91 \%$ of the SARS-CoV-19-specialized practices and by $12.85 \%$ of the non-specialized practices. SARS-CoV19-specialized practices were significantly more often satisfied by their dental associations than those not classified as specialized practices $(p=0.001)$. To prevent SARS-CoV-19 spread, $25.42 \%$ of the private practices worked in separate teams in a shift system and $12.92 \%$ worked in separate teams without a shift system. Of these, $27.92 \%$ took other measures and $36.67 \%$ did not take any additional measures to address this point.

Detailed key economic figures were only available from private practices in this study. Turnover for medical and dental procedures billed to public insurance had reduced to $58.81 \%$ and $62.43 \%$, respectively, of the corresponding values for 2019. Similarly, procedures billed to private insurers or directly to the patient had reduced to $55.17 \%$ of the turnover in 2019 . The majority $(58.75 \%)$ of private practices applied for shorttime allowances from the state, while $41.25 \%$ did not apply for these allowances. The short-time allowances were approved for $39.17 \%$ of the practices, while $19.58 \%$ of the private practices had not received the money despite applying for it.

Among the private practices, $14.71 \%$ reported contact with SARS-CoV-19-positive patients, although only $10.83 \%$ of the practices had been classified as SARS-CoV-19-specialized practices. These specialized practices received SARS-CoV19-positive patients significantly more often $(p=0.017)$.

Hospitals were asked about the surgical procedures conducted in April and the surgical procedures that were planned to be conducted from May: oncologic procedures $(98.15 \%$ in April vs $98.11 \%$ from May), trauma care $(100.00 \%$ vs $100.00 \%)$, and surgical treatment of infections $(100.00 \%$ vs $98.11 \%$ ) were hardly affected by the changes in the 
Table 1 Contents of the questionnaires for (non-) university hospitals (left) and private practices (right). FASHI - Federal Association of Statutory Health Insurance Physicians, FASHD - Federal Association of Statutory Health Insurance Dentists.

\begin{tabular}{|c|c|}
\hline Question & Possible answers \\
\hline \multicolumn{2}{|l|}{ Hospitals } \\
\hline Federal state of the participant & Every German federal state was available for selection \\
\hline Infrastructure & University hospital/non-university hospital \\
\hline $\begin{array}{l}\text { Percentage of ward capacity used compared to that before the } \\
\text { pandemic }\end{array}$ & Freely selectable number \\
\hline $\begin{array}{l}\text { Percentage of current outpatient surgical procedures with local } \\
\text { anesthesia compared to that before the pandemic }\end{array}$ & Freely selectable number \\
\hline $\begin{array}{l}\text { Percentage of current outpatient surgical procedures with general } \\
\text { anesthesia compared to that before the pandemic }\end{array}$ & Freely selectable number \\
\hline $\begin{array}{l}\text { Percentage of current inpatient surgical procedures compared to } \\
\text { that before the pandemic }\end{array}$ & Freely selectable number \\
\hline Do you routinely conduct SARS-CoV-19 screening tests? & No/once preoperatively/twice preoperatively/before admission / after admission \\
\hline $\begin{array}{l}\text { PPE used for surgical procedures for patients assumed to be } \\
\text { SARS-CoV-19-negative }\end{array}$ & Surgical mask/FFP2 respirator/FFP3 respirator/3D-printed face shield \\
\hline Types of surgical procedures currently conducted & $\begin{array}{l}\text { Oncologic procedures/trauma care/surgical treatment of infections/osteonecrosis/ } \\
\text { orthognathic surgery/cleft surgery/bone augmentations/dental implants/- } \\
\text { dentoalveolar procedures such as comprehensive dental treatment under genera } \\
\text { anesthesia }\end{array}$ \\
\hline $\begin{array}{l}\text { Types of surgical procedures planned to be conducted by } \\
\text { May } 2020\end{array}$ & $\begin{array}{l}\text { Oncologic procedures/trauma care/surgical treatment of infections/- } \\
\text { osteonecrosis/orthognathic surgery/cleft surgery/bone augmentations/dental } \\
\text { implants/dentoalveolar procedures such as comprehensive dental treatment } \\
\text { under general anesthesia }\end{array}$ \\
\hline \multicolumn{2}{|l|}{ Private practices } \\
\hline Federal state of the participant & Every German federal state was available for selection \\
\hline Is your office a SARS-CoV-19-specialized practice? & Yes/no \\
\hline Change in patient contacts compared to 2019 & Freely selectable number \\
\hline Sufficiency of supply of PPE by FASFD and FASHI & $\begin{array}{l}\text { Sufficient by FASFD/not sufficient by FASFD/sufficient by FASHI/not sufficien } \\
\text { by FASHI }\end{array}$ \\
\hline Have you applied for short-time allowances & Applied for and approved/applied for \& not (yet) approved/not applied for \\
\hline Measures taken to prevent SARS-CoV-19 spread within the office & $\begin{array}{l}\text { Work in separate teams in a shift system/Work in separate teams without a shift } \\
\text { system/other measures/no additional measures }\end{array}$ \\
\hline $\begin{array}{l}\text { Change in the number of outpatient surgical procedures with local } \\
\text { anesthesia compared to } 2019\end{array}$ & Freely selectable number \\
\hline $\begin{array}{l}\text { Change in the number of outpatient surgical procedures with } \\
\text { general anesthesia compared to } 2019\end{array}$ & Freely selectable number \\
\hline Do you have beds available at a hospital for inpatients? & Yes/no \\
\hline $\begin{array}{l}\text { Change in the number of inpatient surgical procedures compared } \\
\text { to } 2019\end{array}$ & Freely selectable number \\
\hline Change in the turnover billed to public and private insurance & Freely selectable numbers \\
\hline Did you have contact with SARS-CoV-19-positive patients? & Yes/no \\
\hline Do you routinely conduct SARS-CoV-19 screening tests? & No/once preoperatively/twice preoperatively \\
\hline PPE used for surgical procedures & Surgical mask/FFP2 respirator/FFP3 respirator/3D-printed face shield \\
\hline $\begin{array}{l}\text { Types of surgical procedures currently conducted and procedures } \\
\text { planned to be conducted from June } 2020 \text { onwards }\end{array}$ & $\begin{array}{l}\text { Dentoalveolar/skin tumors/traumatology/surgical treatment of } \\
\text { infections/osteonecrosis/orthognathic surgery/bone augmentations/dental } \\
\text { implants/comprehensive dental treatment under general anesthesia }\end{array}$ \\
\hline
\end{tabular}

regulations by the federal ministry of health. Surgical procedures for the treatment of osteonecrosis $(85.19 \%$ vs $96.23 \%)$ were rescheduled in some hospitals. However, the schedules for orthognathic surgery (14.81\% vs $58.49 \%)$, cleft surgery (44.44\% vs $67.92 \%)$, bone augmentation (11.11\% vs $37.74 \%$ ), dental implants (18.52\% vs $50.94 \%)$, and other dentoalveolar procedures such as comprehensive dental treatment under general anesthesia $(59.26 \%$ vs $79.25 \%)$ were subject to major changes.

Due to the differences in regulations and official recommendations and the differences in the surgical spectrum, private practices were provided with a different questionnaire. 
Fig. 1 Conduct of routine SARSCoV-19 PCR tests
Routine SARS-CoV-19 PCR test

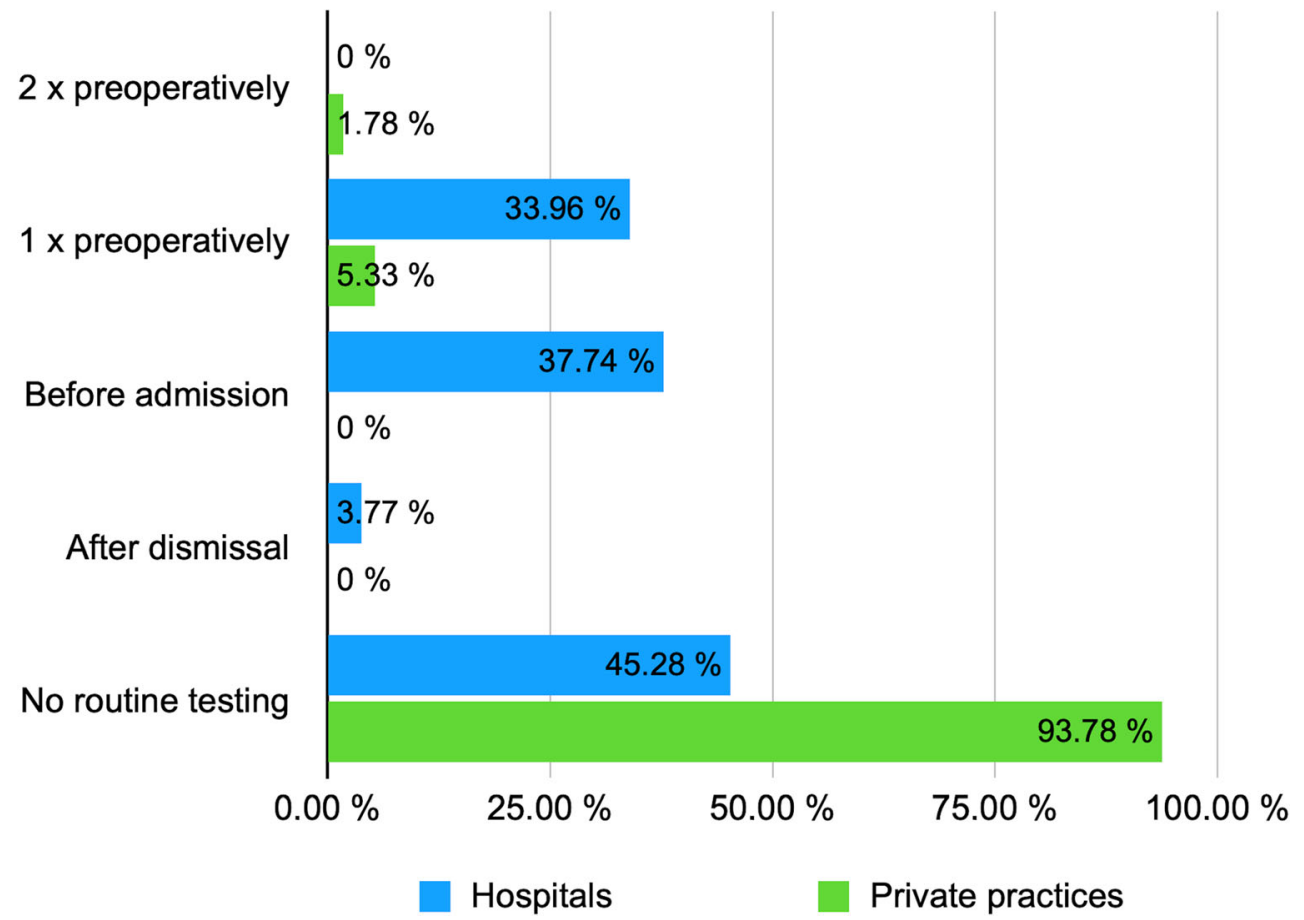

They were asked to compare their surgical procedures planned to be conducted in April 2020 to those planned from June 2020. Dermal oncologic procedures (79.66\% in April 2020 vs $91.10 \%$ planned from June 2020), dentoalveolar procedures $(96.19 \%$ vs $99.15 \%)$, surgical treatment of infections ( $86.44 \%$ vs $85.59 \%$ ), and comprehensive dental treatment under general anesthesia $(75.00 \%$ vs $87.29 \%)$ were hardly affected by the SARS-CoV-19 situation. Some changes were seen in the scheduling of trauma care $(22.88 \%$ vs $33.47 \%)$ and surgical procedures for the treatment of osteonecrosis (48.31\% vs $69.07 \%$ ). However, orthognathic surgeries (2.54\% vs $17.37 \%)$, bone augmentation procedures $(57.20 \%$ vs $92.80 \%$ ), and dental implant procedures $(71.61 \%$ vs $97.03 \%$ ) were subject to major changes.
Fig. 2 Use of personal protection equipment (PPE) for patients not known to be SARS-CoV-19positive

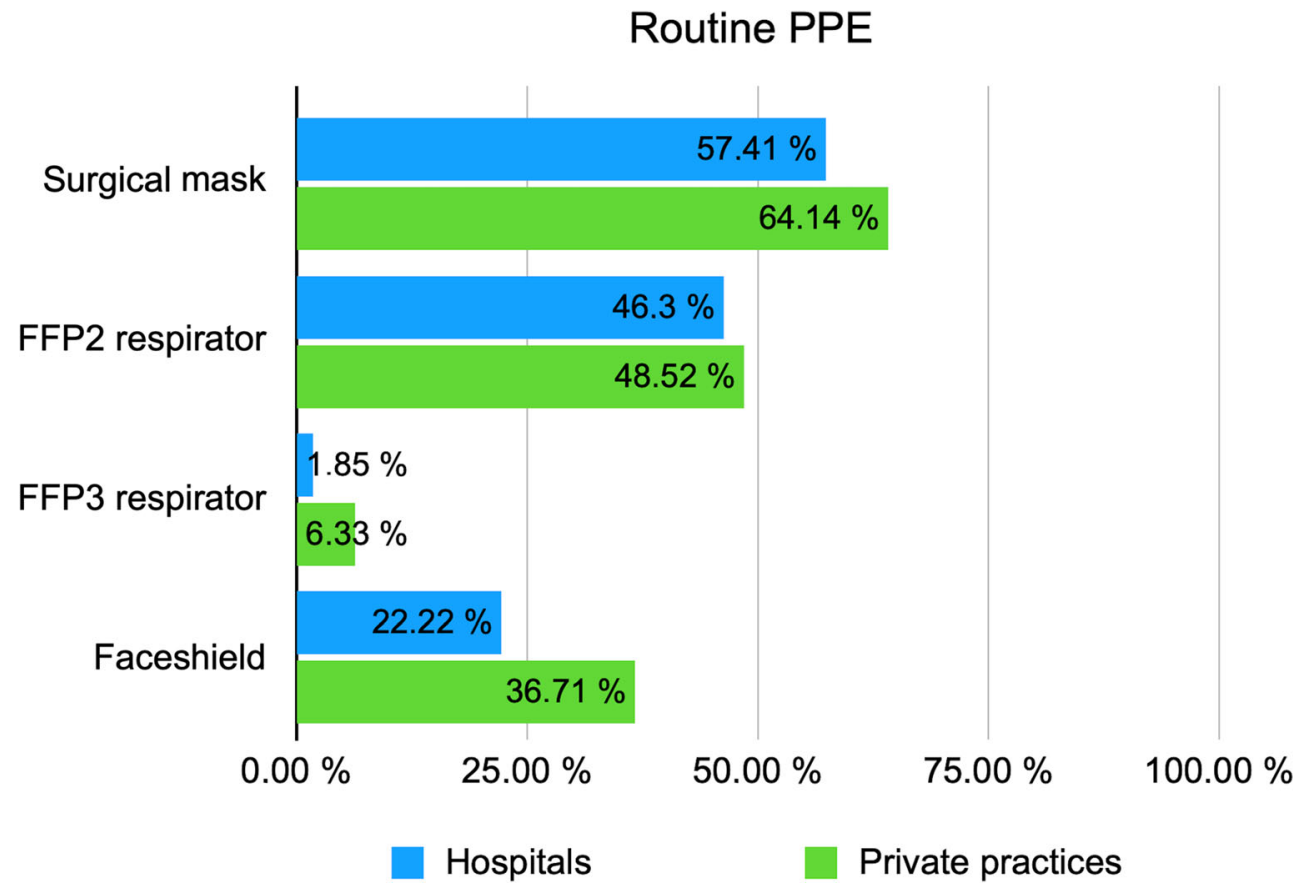




\section{Discussion}

The SARS-CoV-2 pandemic has significantly decreased the number of patients and the number of surgical procedures in all surgical specialties [21]. In the vascular surgery division in the USA the weekly clinic volume and surgical volume reduced by $96.5 \%$ and $71.1 \%$, respectively [22]. The extent of these reductions is dependent on different aspects, including the surgical specialty, hospital, and country. During the SARS-CoV-2 pandemic, the conduct of essential and non-deferrable operations such as tumor surgery was challenging while elective operations were postponed [23]. In this context, Maffia et al. conducted a global survey of 154 surgical centers for oral and maxillofacial surgery in 54 countries to assess their current practices in oral and maxillofacial surgery [24]. Despite the differences in oral and maxillofacial practice in Germany from those in other countries, the results were found to be similar to those presented herein. Maffia et al. demonstrated that the three most required sub-specialties in oral and maxillofacial surgery during the pandemic were-without a more accurate breakdown - (1) traumatology, (2) oncologic surgery, and (3) oral surgery. The reported outbreak activity indices (OAIs) of $83.2 \%, 66.7 \%$, and $38.3 \%$, respectively, are consistent with the findings of the current study [24]. Similar results were reported for dentistry. The number of patients was significantly reduced, while the number of dental infections significantly increased. In addition, the frequency of dental trauma decreased [17]. One possible explanation for the decreased frequency of dental trauma might be the contact bans and curfews in some countries resulting in reduced sports and leisure activities, such as (team-) sports that are associated with an increased risk for dental trauma. The most comprehensive restrictions were seen in orthognathic surgery (OAI, 8.7\%) and temporomandibular joint (TMJ) surgery (OAI, 6.9\%). Next, the study by Maffia et al. reported that 28 departments of oral and maxillofacial surgery were completely closed, of which 7 were located in European countries, and 6 departments reported to work regularly without any restrictions during the pandemic [24]. Subsequently, the SARS-CoV-2 pandemic also influenced oral and maxillofacial surgery training and education [25].

The findings of the study clearly show that the frequencies of orthognathic surgery, cleft surgery, and dental implant placement were taken up again at the hospitals from April to May 2020, as the situation improved. The reduced bed capacities and thus SARS-CoV-2-induced financial losses in hospitals were to some degree financially directly compensated for by the government. This was achieved by direct payments for building the reserve capacity of intensive care beds. Logically, this reduced the capacities for elective procedures. In contrast, private practices were unable to receive such compensation to improve intensive care capacities. For private practices, especially those performing dental implant placement and augmentation procedures, the number of surgical interventions increased sharply from April to June 2020. One reason for this increase might be the backlog of patients requiring implant and augmentation procedures and the fact that these patients were unable to receive treatment in hospitals because of the aforementioned intensive care capacity regulations. This observation can also be explained by the different range of treatments in private practices, which generally focus more on dental implantology than hospitals.

Costa et al. performed a nationwide survey of 142 oral and maxillofacial surgeons in Brazil [26]. Contrary to the findings of the present study, the results of Costa et al. illustrated a lack of knowledge regarding different aspects of SARS-CoV-2, such as transmission routes, symptoms, laboratory findings, hygiene, and infection prevention, which might be considered as one of the public health problems responsible for the high infection rates in Brazil [26].

The results of our study demonstrate an extremely low number of SARS-CoV-2 screening tests performed in hospitals and private practices. Currently, testing methods for SARS-CoV-2 virus (RT-PCR), SARS-CoV-2 antibody (e.g., ELISA), and SARS-CoV-2 antigen detection are available [27-29]. Unfortunately, even the most sensitive and specific SARS-CoV-2 tests are associated with a significant ratio of false-positive tests [30]. One reason for the low testing rates in Germany might be the lack of detailed recommendations regarding SARS-CoV-2 tests, especially for private practices. Unfortunately, the exact reasons for the low frequency of SARS-CoV-2 tests could not be captured in detail in this survey. This might be reserved for a subsequent study.

In comparison to other countries, Germany and Europe lack available guidelines for the treatment and management of patients requiring oral and maxillofacial surgery [31]. To focus hygiene measures to the point of need, some oral and maxillofacial surgery practices in Germany have been assigned as SARS-CoV-2-specialized practices to treat SARS-CoV-2-infected patients. These specialized practices have been equipped with more PPE and served as contact points for SARS-CoV-2-infected patients requiring oral and maxillofacial treatment, with participation from the Federal Association of Statutory Health Insurance Physicians and the Federal Association of Statutory Health Insurance Dentists. Besides that, workflows in hospitals and private practices have had to be re-organized in order to prevent cross-sectional infections among patients. To accomplish this, significant changes in the infrastructure of in- and outpatient units and operating rooms have had to be implemented [32].

With respect to the economic burden of the SARS-CoV-2 pandemic on dentistry in Germany, utilization of all dental services has been significantly reduced $(-80 \%$ prevention, $76 \%$ periodontics, $-70 \%$ prosthetics) [33]. In comparison to the decrease in the patient and surgery numbers in oral and maxillofacial surgery, the corresponding reductions in the field of dentistry might be higher, since general dental practice 
includes a higher proportion of elective procedures than oral and maxillofacial surgery. In this regard, some hospitals and dental practices may not survive the pandemic or may not be able to restart general practice after the pandemic [34].

During the SARS-CoV-2 pandemic, technologies such as telemedicine have become increasingly popular to ensure patient care and prevent SARS-CoV-2 infections [35]. This change can be seen as an opportunity to include these concepts in oral and maxillofacial practice as well. In addition, there is a need for national guidelines for medical care related to oral and maxillofacial surgery during exceptional situations such as pandemics. These guidelines should be established in accordance with the experiences during the SARS-CoV-2 pandemic, even though the there is still a lack of data availability regarding this by now [36]. Patel et al. designated the pandemic as an opportunity for oral and maxillofacial surgery to be acknowledged in medicine and for oral and maxillofacial surgeons to take frontline roles and use their wide-ranging skills gained from training in oral and maxillofacial surgery [37]. Finally, the patient care can be considered well-adapted to the SARS-CoV-2 pandemic in the field of oral and maxillofacial surgery. Even there was a reduction of surgical procedures of in- and outpatients in OMFS, there is a necessity to provide adequate surgical capacities in OMFS to ensure OMFS patient care, on the one hand in the fields of oncology, traumatology and septic surgery and on the other hand in further OMFS areas such as primary malformation surgery.

However, this study had some limitations. Both online surveys were completed at different time points, and both surveys were open for completion for days. Due to the rapid developments during that time, the significantly differing external influences between the participants might have caused bias. Furthermore, the private practices were necessarily contacted indirectly by sending invitations to all DGMKG members working in this sector. Thus, we cannot entirely rule out the possibility that some participants may not have followed the instruction to complete one questionnaire per private practice. Even though participation was voluntary and the evaluation was carried out anonymously, the influence of personal interests when answering these questions cannot be fully elucidated.

\section{Conclusions}

For oral and maxillofacial surgery, the German healthcare system has shown the ability to adapt in- and outpatient care to emergency situations such as the SARS-CoV-2 pandemic. Patient care in oral and maxillofacial surgery has been ensured at hospitals and private practices in more difficult conditions during the pandemic. Adequate surgical capacities for in- and outpatients care are essentially and have to be ensured in OMFS even in exceptional circumstances such as SARSCoV-2 pandemic. However, the extent of the immense economic burden imposed by the pandemic on hospitals and private practices cannot be conclusively assessed at this point.

Acknowledgements We would like to thank all participants of the survey, the board, and all members and colleagues of the German Association of Oral and Maxillofacial Surgery (DGMKG) for supporting this survey.

\section{Compliance with ethical standards}

Conflict of interest The authors declare that they have no conflict of interest.

Ethical approval No ethical approval was required. This article is based on a voluntary, anonymously evaluated online survey. From the results presented, individual participants could not be tracked down by their answers given. The study did not include any animal studies performed by any of the authors.

Informed consent For this type of study, formal consent is not required.

\section{References}

1. Lai CC, Shih TP, Ko WC, Tang HJ, Hsueh PR (2020) Severe acute respiratory syndrome coronavirus 2 (SARS-CoV-2) and coronavirus disease-2019 (COVID-19): the epidemic and the challenges. Int J Antimicrob Agents 55(3):105924. https://doi.org/10.1016/j. ijantimicag.2020.105924

2. Saggese NP, Cardo VA (2020) A perspective from a NYC chief oral and maxillofacial surgery resident during the COVID-19 pandemic. Br J Oral Maxillofac Surg. 58:730-731. https://doi.org/10. 1016/j.bjoms.2020.04.042

3. Fu Y, Cheng Y, Wu Y (2020) Understanding SARS-CoV-2mediated inflammatory responses: from mechanisms to potential therapeutic tools. Virol Sin 35(3):266-271. https://doi.org/10. 1007/s12250-020-00207-4

4. Zheng Z, Peng F, Xu B, Zhao J, Liu H, Peng J, Li Q, Jiang C, Zhou Y, Liu S, Ye C, Zhang P, Xing Y, Guo H, Tang W (2020) Risk factors of critical \& mortal COVID-19 cases: a systematic literature review and meta-analysis. J Infect. 81:e16-e25. https://doi.org/10. 1016/j.jinf.2020.04.021

5. Ackermann M, Verleden SE, Kuehnel M, Haverich A, Welte T, Laenger F, Vanstapel A, Werlein C, Stark H, Tzankov A, Li WW, Li VW, Mentzer SJ, Jonigk D (2020) Pulmonary vascular endothelialitis, thrombosis, and angiogenesis in Covid-19. N Engl J Med 383(2):120-128. https://doi.org/10.1056/NEJMoa2015432

6. Remuzzi A, Remuzzi G (2020) COVID-19 and Italy: what next? Lancet 395(10231):1225-1228. https://doi.org/10.1016/S01406736(20)30627-9

7. Kowalski LP, Sanabria A, Ridge JA, Ng WT, de Bree R, Rinaldo A, Takes RP, Makitie AA, Carvalho AL, Bradford CR, Paleri V, Hartl DM, Vander Poorten V, Nixon IJ, Piazza C, Lacy PD, Rodrigo JP, Guntinas-Lichius O, Mendenhall WM, D'Cruz A, Lee AWM, Ferlito A (2020) COVID-19 pandemic: effects and evidence-based recommendations for otolaryngology and head and neck surgery practice. Head Neck 42(6):1259-1267. https:// doi.org/10.1002/hed.26164

8. Zeng L, Su T, Huang L (2020) Strategic plan for management in oral and maxillofacial surgery during COVID-19 epidemic. Oral Oncol 105:104715. https://doi.org/10.1016/j.oraloncology.2020. 104715 
9. Sabino-Silva R, Jardim ACG, Siqueira WL (2020) Coronavirus COVID-19 impacts to dentistry and potential salivary diagnosis. Clin Oral Investig 24(4):1619-1621. https://doi.org/10.1007/ s00784-020-03248-x

10. Thamboo A, Lea J, Sommer DD, Sowerby L, Abdalkhani A, Diamond C, Ham J, Heffernan A, Cai Long M, Phulka J, Wu YQ, Yeung P, Lammers M (2020) Clinical evidence based review and recommendations of aerosol generating medical procedures in otolaryngology - head and neck surgery during the COVID-19 pandemic. J Otolaryngol Head Neck Surg 49(1):28. https://doi. org/10.1186/s40463-020-00425-6

11. Day AT, Sher DJ, Lee RC, Truelson JM, Myers LL, Sumer BD, Stankova L, Tillman BN, Hughes RS, Khan SA, Gordin EA (2020) Head and neck oncology during the COVID-19 pandemic: reconsidering traditional treatment paradigms in light of new surgical and other multilevel risks. Oral Oncol 105:104684. https://doi. org/10.1016/j.oraloncology.2020.104684

12. Diegritz C, Manhart J, Bucher K, Grabein B, Schuierer G, Kuhnisch J, Kunzelmann KH, Hickel R, Fotiadou C (2020) A detailed report on the measures taken in the Department of Conservative Dentistry and Periodontology in Munich at the beginning of the COVID-19 outbreak. Clin Oral Investig 24(8):29312941. https://doi.org/10.1007/s00784-020-03440-z

13. Zhao Z, Gao D (2020) Precaution of 2019 novel coronavirus infection in department of oral and maxillofacial surgery. Br J Oral Maxillofac Surg 58(3):250-253. https://doi.org/10.1016/j.bjoms. 2020.03.001

14. Panesar K, Dodson T, Lynch J, Bryson-Cahn C, Chew L, Dillon J (2020) Evolution of COVID-19 Guidelines for University of Washington Oral and Maxillofacial Surgery Patient Care. J Oral Maxillofac Surg 78(7):1136-1146. https://doi.org/10.1016/j.joms. 2020.04.034

15. Givi B, Schiff BA, Chinn SB, Clayburgh D, Iyer NG, Jalisi S, Moore MG, Nathan CA, Orloff LA, O'Neill JP, Parker N, Zender C, Morris LGT, Davies L (2020) Safety recommendations for evaluation and surgery of the head and neck during the COVID-19 pandemic. JAMA Otolaryngol Head Neck Surg. 146:579-584. https://doi.org/10.1001/jamaoto.2020.0780

16. Blackhall KK, Downie IP, Ramchandani P, Kusanale A, Walsh S, Srinivasan B, Shields H, Brennan PA, Singh RP (2020) Provision of emergency maxillofacial service during the COVID-19 Pandemic: a Collaborative Five Centre UK Study. Br J Oral Maxillofac Surg. 58:698-703. https://doi.org/10.1016/j.bjoms. 2020.05.020

17. Guo H, Zhou Y, Liu X, Tan J (2020) The impact of the COVID-19 epidemic on the utilization of emergency dental services. J Dent Sci. https://doi.org/10.1016/j.jds.2020.02.002

18. Yang Y, Zhou Y, Liu X, Tan J (2020) Health services provision of 48 public tertiary dental hospitals during the COVID-19 epidemic in China. Clin Oral Investig 24(5):1861-1864. https://doi.org/10. 1007/s00784-020-03267-8

19. Ishack S, Lipner SR (2020) Applications of 3D printing technology to address COVID-19-related supply shortages. Am J Med 133(7): 771-773. https://doi.org/10.1016/j.amjmed.2020.04.002

20. Swennen GRJ, Pottel L, Haers PE (2020) Custom-made 3D-printed face masks in case of pandemic crisis situations with a lack of commercially available FFP2/3 masks. Int J Oral Maxillofac Surg 49(5):673-677. https://doi.org/10.1016/j.ijom.2020.03.015

21. Latz CA, Boitano LT, Png CYM, Tanious A, Kibrik P, Conrad M, Eagleton M, Dua A (2020) Early vascular surgery response to the COVID-19 pandemic: results of a nationwide survey. J Vasc Surg. https://doi.org/10.1016/j.jvs.2020.05.032

22. Hemingway JF, Singh N, Starnes BW (2020) Emerging practice patterns in vascular surgery during the COVID-19 pandemic. J Vasc Surg. 72:396-402. https://doi.org/10.1016/j.jvs.2020.04.492
23. Valentini V, Pucci R, Battisti A, Cassoni A (2020) Head and neck cancer cannot wait for this pandemic to end: risks, challenges and perspectives of oral-maxillofacial surgeon during COVID-19. Oral Oncol 106:104758. https://doi.org/10.1016/j.oraloncology.2020. 104758

24. Maffia F, Fontanari M, Vellone V, Cascone P, Mercuri LG (2020) Impact of COVID-19 on maxillofacial surgery practice: a worldwide survey. Int J Oral Maxillofac Surg 49(6):827-835. https://doi. org/10.1016/j.ijom.2020.04.015

25. Huntley RE, Ludwig DC, Dillon JK (2020) Early effects of COVID-19 on oral and maxillofacial surgery residency trainingresults from a National Survey. J Oral Maxillofac Surg. 78:12571267. https://doi.org/10.1016/j.joms.2020.05.026

26. Costa SM, Lacerda GT, Villafort RN, Silveira RL, Amaral MBF (2020) We know about COVID-19: Oral and Maxillofacial Surgeons Survey. J Craniofac Surg. https://doi.org/10.1097/SCS. 0000000000006658 Publish Ahead of Print

27. Hong KH, Lee SW, Kim TS, Huh HJ, Lee J, Kim SY, Park JS, Kim GJ, Sung H, Roh KH, Kim JS, Kim HS, Lee ST, Seong MW, Ryoo N, Lee H, Kwon KC, Yoo CK (2020) Guidelines for laboratory diagnosis of coronavirus disease 2019 (COVID-19) in Korea. Ann Lab Med 40(5):351-360. https://doi.org/10.3343/alm.2020.40.5. 351

28. Grenache DG, Sever C, Mathur G, Mathur S (2020) Antibody testing for COVID-19. Am J Clin Pathol. 154:425-426. https:// doi.org/10.1093/ajcp/aqaa110

29. Scohy A, Anantharajah A, Bodeus M, Kabamba-Mukadi B, Verroken A, Rodriguez-Villalobos H (2020) Low performance of rapid antigen detection test as frontline testing for COVID-19 diagnosis. J Clin Virol 129:104455. https://doi.org/10.1016/j.jcv. 2020.104455

30. Kumleben N, Bhopal R, Czypionka T, Gruer L, Kock R, Stebbing J, Stigler FL (2020) Test, test, test for COVID-19 antibodies: the importance of sensitivity, specificity and predictive powers. Public Health 185:88-90. https://doi.org/10.1016/j.puhe.2020.06.006

31. Yang Y, Soh HY, Cai ZG, Peng X, Zhang Y, Guo CB (2020) Experience of diagnosing and managing patients in oral maxillofacial surgery during the prevention and control period of the new coronavirus pneumonia. Chin J Dent Res 23(1):57-62. https://doi. org/10.3290/j.cjdr.a44339

32. Zimmermann M, Nkenke E (2020) Approaches to the management of patients in oral and maxillofacial surgery during COVID-19 pandemic. J Craniomaxillofac Surg 48(5):521-526. https://doi. org/10.1016/j.jcms.2020.03.011

33. Schwendicke F, Krois J, Gomez J (2020) Impact of SARS-CoV2 (Covid-19) on dental practices: economic analysis. J Dent:103387. https://doi.org/10.1016/j.jdent.2020.103387

34. Ferneini EM (2020) The financial impact of COVID-19 on our practice. J Oral Maxillofac Surg 78(7):1047-1048. https://doi.org/ 10.1016/j.joms.2020.03.045

35. Giudice A, Barone S, Muraca D, Averta F, Diodati F, Antonelli A, Fortunato L (2020) Can teledentistry improve the monitoring of patients during the Covid-19 dissemination? a descriptive pilot study. Int J Environ Res Public Health 17(10). https://doi.org/10. 3390/ijerph17103399

36. Cirillo N (2020) COVID-19 outbreak: succinct advice for dentists and oral healthcare professionals. Clin Oral Investig 24(7):2529 2535. https://doi.org/10.1007/s00784-020-03323-3

37. Patel NA, Ji YD, Odera SL (2020) The role of oral and maxillofacial surgeons in COVID-19 response. J Oral Maxillofac Surg 78(7): 1052-1053. https://doi.org/10.1016/j.joms.2020.04.016

Publisher's note Springer Nature remains neutral with regard to jurisdictional claims in published maps and institutional affiliations. 